\title{
Observation and simulations of winds and temperatures in the Antarctic thermosphere for August 2-10, 1992
}

\author{
R. W. Smith, ${ }^{1}$ G. Hernandez, ${ }^{2}$ R. G. Roble, ${ }^{3}$ P. L. Dyson, ${ }^{4}$ M. Conde, ${ }^{1}$ R. Crickmore, ${ }^{5}$ \\ and M. Jarvis ${ }^{6}$
}

\begin{abstract}
Optically derived upper thermospheric wind and temperature data, collected at Antarctic stations at South Pole $(L=14)$, Mawson $(L=9.3)$, and Halley $(L=4.6)$, and averaged over the low-activity period August 2-10, 1992, have been interpreted with the help of simulation by the National Center for Atmospheric Research thermosphere ionosphere electrodynamic general circulation model (TIEGCM) with inputs matching the average conditions of observation. The simulation provides a global background context upon which the widely-separated optical observations can be placed. The simulation shows three large-scale structures in the polar wind field: the morning vortex, the evening vortex, and the cross-polar wind jet. Each of these came within view of the group of observing stations during the diurnal cycle, providing arrival time observations and signatures which were examined relative to the TIEGCM simulation. Reasonable correspondence was found, indicating the capability of the model to agree simultaneously with observations at three widely spaced stations representative of the subauroral and auroral zones, as well as the polar cap. Simulated wind directions were in excellent agreement with observation, although wind magnitudes frequently exceeded measured values by up to $30 \%$. Apparent divergent flows in the data from Halley and Mawson were explained as signatures of vortices from their presence in the simulated wind fields. Observed diurnal mean temperatures compared well with the simulation, confirming that heat inputs and the distribution of thermal energy in the model are, on average, reasonable. A significant and persistent difference between experimental and modeled temperatures was that the diurnal temperature variation observed at South Pole peaked at the nightside crossing of the jet and was minimum a few hours before noon magnetic local time, whereas the simulation indicated minimum temperatures on the nightside, in antiphase to the measurements. A simple calculation indicates that the observed temperature difference between the air parcels entering the polar cap, encountered on the dayside, and those leaving the polar cap on the nightside is reasonably matched to the heating due to the ion-drag acceleration process. No explanation of the lack of this temperature rise in the TIEGCM simulation is presently available.
\end{abstract}

\section{Introduction}

In recent years, several studies [Crowley et al., 1989; Smith et al., 1989; Codrescu et al., 1992; Roble, 1992; Forbes et al., 1993; Deng et al., 1993; Lu et al., 1995; Burns et al., 1995; Hernandez and Roble, 1995] have shown the general success of thermospheric simulation by thermosphere ionosphere electrodynamic general circulation models (TIGCMs). Comparisons with data from single stations and multiple stations have shown that many of the features of the observed diurnal, seasonal, and geomagnetic variations can be correctly modeled. Nevertheless, there remain some significant differences between data and models which require investigation to determine whether or not there are yet major factors that may still

\footnotetext{
'Geophysical Institute, University of Alaska, Fairbanks.

${ }^{2}$ Graduate Program in Geophysics, University of Washington, Seattle.

${ }^{3}$ High Altitude Observatory, National Center for Atmospheric Research, Boulder, Colorado.

${ }^{4}$ School of Physics, LaTrobe University, Bundoora, Victoria, Australia. ${ }^{\text {s} W i n f r i t h ~ T e c h n o l o g y ~ C e n t r e, ~ D o r c h e s t e r, ~ E n g l a n d, ~ U n i t e d ~ K i n g d o m . ~}$ ${ }^{6} B$ ritish Antarctic Survey, Cambridge, England, United Kingdom.
}

Copyright 1998 by the American Geophysical Union.

Paper number 97JA03336.

0148-0227/98/97JA-03336\$09.00 require inclusion in the simulation code. Recent work [Sojka et al., 1988; Rishbeth et al., 1995] has shown that there is still progress to be made in correctly modeling $f_{0} F_{2}$ and $h m F_{2}$. These are easily measured properties of the $F$ region ionosphere, but their values are critically dependent on some coupling processes between the ionosphere and thermosphere caused by the in situ wind, advection of chemically modified air parcels from high latitudes, and changes in thermospheric temperature. One of the critical aspects of studies related to imperfectly modeled ionosphere-thermosphere coupling is the neutral wind field. A recent improvement to the National Center for Atmospheric Research (NCAR) TIGCM, called the TIEGCM (thermosphere ionosphere electrodynamic GCM) [Richmond et al., 1992], now includes self-consistently calculated polarization electric fields. The effects of ion-neutral coupling should now be more refined and more correct, in particular where externally imposed electric fields are small.

This study addresses the agreement between observation and simulation of winds and temperatures in the upper thermosphere by the comparison of data from three Antarctic stations with a TIEGCM simulation. We chose a data period for which geomagnetic activity was low and $F 10.7$ was moderate. In the next section we describe the simultaneously recorded data. Following that, the results of the TIEGCM runs are presented and discussed in relation to the measurements. 
Table 1. Parameters and Data from Observing Stations

\begin{tabular}{|c|c|c|c|c|c|}
\hline Station & $\begin{array}{l}\text { Geographic } \\
\text { latitude }\end{array}$ & $\begin{array}{l}\text { Geographic } \\
\text { longitude }\end{array}$ & $\begin{array}{l}\text { Invariant } \\
\text { latitude }\end{array}$ & $\begin{array}{l}\text { Magnetic } \\
\text { midnight }\end{array}$ & $\begin{array}{l}\text { Data } \\
\text { availability }\end{array}$ \\
\hline Halley & -75.52 & 333.32 & -61.62 & 02.73UT & $\begin{array}{l}920802-4 \\
920806-10\end{array}$ \\
\hline Mawson & -67.61 & 62.88 & -70.49 & 22.60UT & $\begin{array}{l}920802-4 \\
920806-10\end{array}$ \\
\hline South Pole & -90.0 & 172.0 & -74.18 & 03.62UT & $920802-10$ \\
\hline
\end{tabular}

Read 920802-4 as August 2, 1992 to August 4, 1992.

\section{Experimental Procedure and Data}

During the period August 2 to August 10, 1992, there were three Fabry-Perot spectrometers in operation in Antarctica making measurements of the wind and temperature of the upper thermosphere. The parameters and data available from each observing station are shown in Table 1. Dates are quoted using the year-month-day (yy, $\mathrm{mm}, \mathrm{dd}$ ) format and in universal time (UT).

The geographical relationship between these stations and the geomagnetic pole is shown in Figure 1 along with the disposition of the auroral oval for $Q=4$ conditions. From this figure it is clear that Halley and South Pole are nearly on the same geomagnetic meridian but separated by 12.5 degrees of magnetic latitude. Mawson, however, is some 5 hours geomagnetic east of South Pole.

Measurements of wind and temperature in the upper thermosphere were made using high-resolution Fabry-Perot spectrometers. The red line $(630 \mathrm{~nm})$ oxygen emission in the aurora and airglow was used as a tracer. At high latitudes the emission altitude may be quite variable from $200 \mathrm{~km}$ in nightside aurora to $350 \mathrm{~km}$ near cusp latitudes. Under conditions of weak geomagnetic disturbance, there is

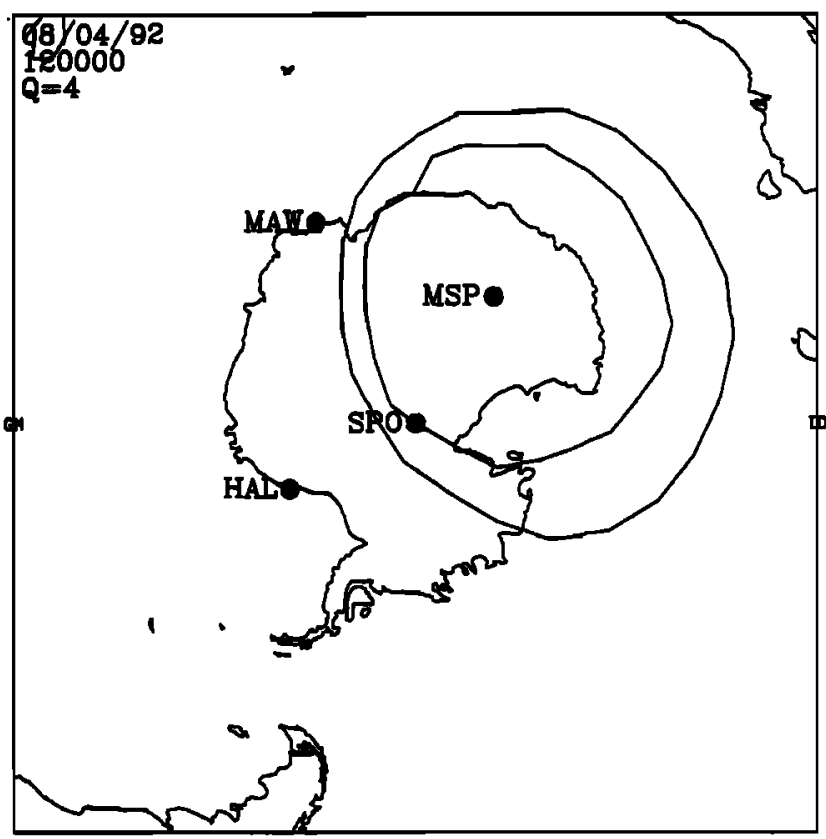

Antarctic Thermospheric Dynanica Stations

Figure 1. Map of Antarctica showing the stations used in this paper: South Pole (SPO, -75.5 Inv), Mawson (MAW, -70.5 Inv) and Halley (HAL, -61.6 Inv). Also marked are the magnetic south pole (MSP) and the auroral oval for $Q=4$ conditions at 1200 UT. little change of horizontal wind with height, and measurements made in the altitude range $200-350 \mathrm{~km}$ can be reasonably combined together to describe upper thermospheric conditions. Operational and instrumental details of the instruments are given by Hernandez et al. [1990] for South Pole, Jacka [1984] for Mawson, and Crickmore et al. [1991] for Halley. Line-of-sight wind observations were reduced to horizontal components in geomagnetic meridional and zonal directions. Eastward and northward winds are shown positive in all the figures in this paper.

Geomagnetic and solar conditions for the chosen days are set out in Table 2. Although the period was quiet as a whole, August 5, 1992 (920805) was excluded since it had an Ap of 35 which was well above the other entries in the table and also since there was a 71-nT negative deflection in Dst. The recovery period in Dst, extending through the next day, caused us to reject 920806 as well for Mawson and South Pole stations, which are at geomagnetically high latitude. The most uniform set of data was found in the period prior to 920805 . In the following description of the experimental data, we will highlight those features of the observations which will be examined in the context of the TIEGCM simulation.

\subsection{South Pole}

Figure 2 shows a plot of meridional and zonal winds in geomagnetic coordinates for the mean of the days 920802 to 920804 . This was the quieter of the two periods either side of 920805 . The meridional wind trace shows observations looking geomagnetically southward (poleward) and northward (equatorward). These observations were separated by about 8 degrees of magnetic latitude. The wind profiles were very similar in shape, with weak poleward flow $(50 \mathrm{~m} / \mathrm{s}$ ) in the dayside sector (geomagnetic noon was 1530 UT) and stronger equatorward flow in the midnight sector. Although there was little difference between the observed poleward flow at $70^{\circ} \mathrm{S}$ and $78^{\circ} \mathrm{S}$ geomagnetic latitude near noon magnetic local time (MLT), there was a substantial decrease in equatorward flow as the air parcels traveled to lower latitudes beginning near magnetic midnight (0330 UT). At the peak of equatorward motion, the meridional wind was about $175 \mathrm{~m} / \mathrm{s}$ at $78^{\circ} \mathrm{S}$ and $150 \mathrm{~m} / \mathrm{s}$ at $70^{\circ} \mathrm{S}$. The zonal wind indicated mostly uniform flow which maximized near 1200 UT at about $75 \mathrm{~m} / \mathrm{s}$. Figure 3, which shows a polar vector plot of the same wind data in magnetic local time, indicates that from magnetic noon to magnetic midnight, the observations at South Pole related to a cross-polar jet approaching the pole and exiting to lower latiudes near 0200 MLT. The major region of light winds from 1700 to 2200 MLT occurred with a rotation of flow westward through poleward to eastward. In the morning sector the winds did not decrease in a similar manner but there was a brief inflection of the mostly westward wind vectors toward poleward.

Table 2.

\begin{tabular}{lrcc}
\hline Date & Ap & Dst (Daily mean value) & F10.7 \\
\hline 920802 & 5 & 2.9 & 128.2 \\
920803 & 4 & 6.4 & 135.1 \\
920804 & 15 & 27.2 & 134.7 \\
920805 & 35 & -44.0 & 134.3 \\
920806 & 16 & -19.3 & 141.9 \\
920807 & 26 & -35.3 & 145.4 \\
920808 & 16 & -38.3 & 147.5 \\
920809 & 12 & -21.2 & 141.4 \\
920810 & 7 & -14.8 & 136.3 \\
\hline
\end{tabular}

Read 920802 as August 2, 1992. 


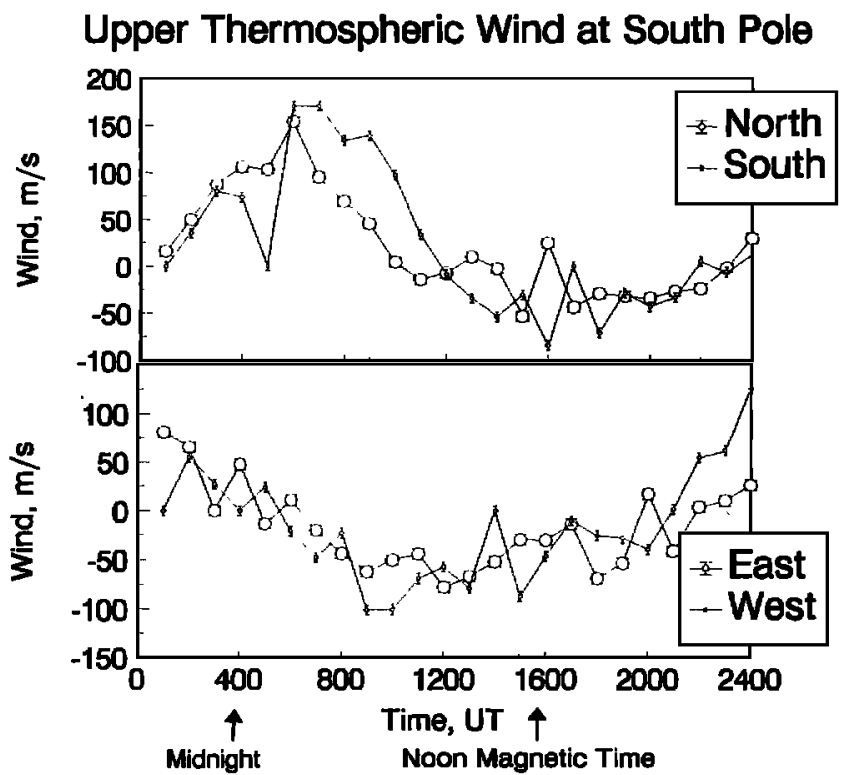

Figure 2. Measured wind components in the upper thermosphere at South Pole station Antarctica averaged for the quiet period 920802 to 920804 (August 2 to 4, 1992).

The upper temperature panel in Figure 4 shows that for South Pole the upper thermosphere had a maximum temperature of $900 \mathrm{~K}$ about 4 hours after magnetic midnight, where the jet departed equatorward for lower latitudes, and $820 \mathrm{~K} 2$ hours before magnetic noon.

\section{Upper Thermospheric Temperatures}

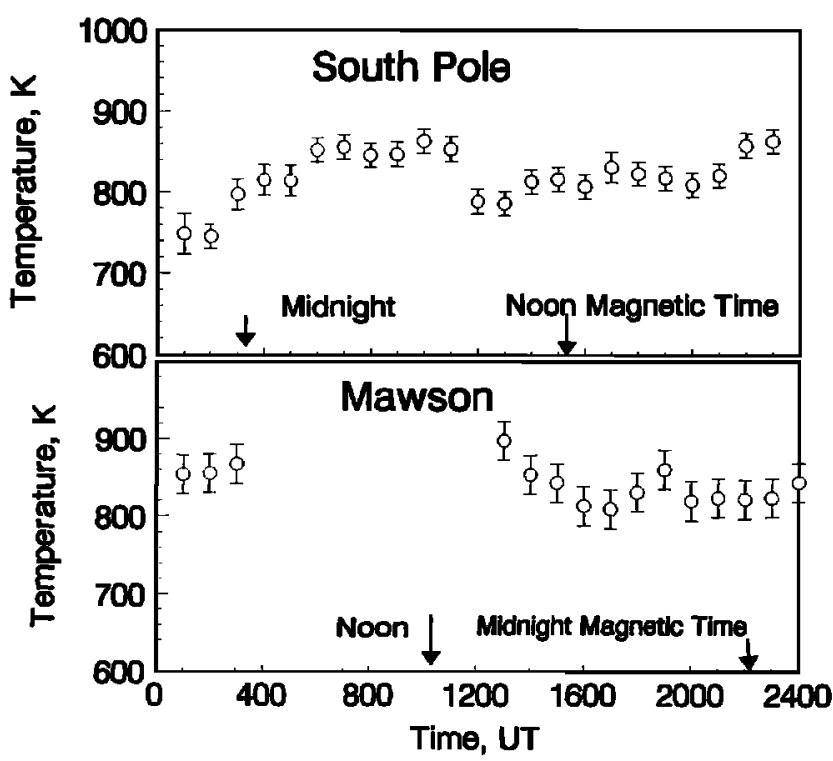

Figure 4. Temperatures in the upper thermosphere measured in the zenith direction at South Pole and Mawson. Averages were made for the quiet period 920802 to 920804 .

\subsection{Mawson}

Geomagnetic meridional and zonal wind components are shown in Figure 5. These were spline-interpolated at half-hour intervals, rotated from the original geographic observed directions, and averaged for the period 920802 to 920804 . A gap occurred in the record when operations were suspended during twilight at daylight. The maximum wind observed in the meridian was $120 \mathrm{~m} / \mathrm{s}$ equatorward, just after magnetic midnight (at $2236 \mathrm{UT}$ ). There was also a persis-

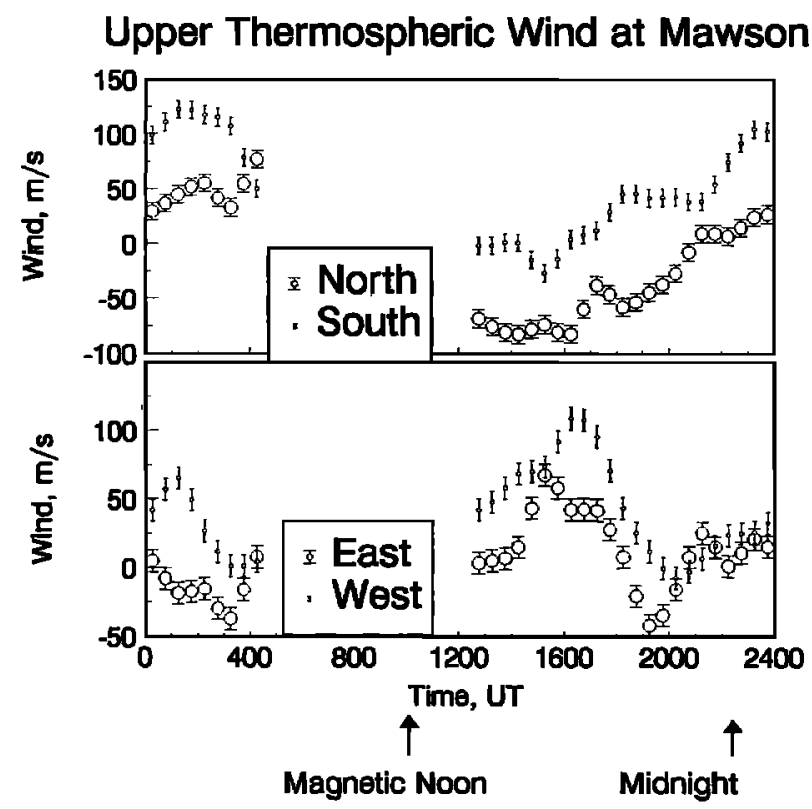

Figure 5. Wind components in the upper thermosphere measured at Mawson, Antarctica, and rotated from observations in the geographic directions and plotted in geomagnetic coordinates. Averages were made for days in the quiet period 920802 to 920804 .
Figure 3. Polar plot of measured winds in the upper thermosphere at South Pole, averaged for the quiet period 920802 to 920804 . The center of this plot is at the geomagnetic pole, the circles are in geomagnetic latitude, and times are marked in magnetic local time (MLT).

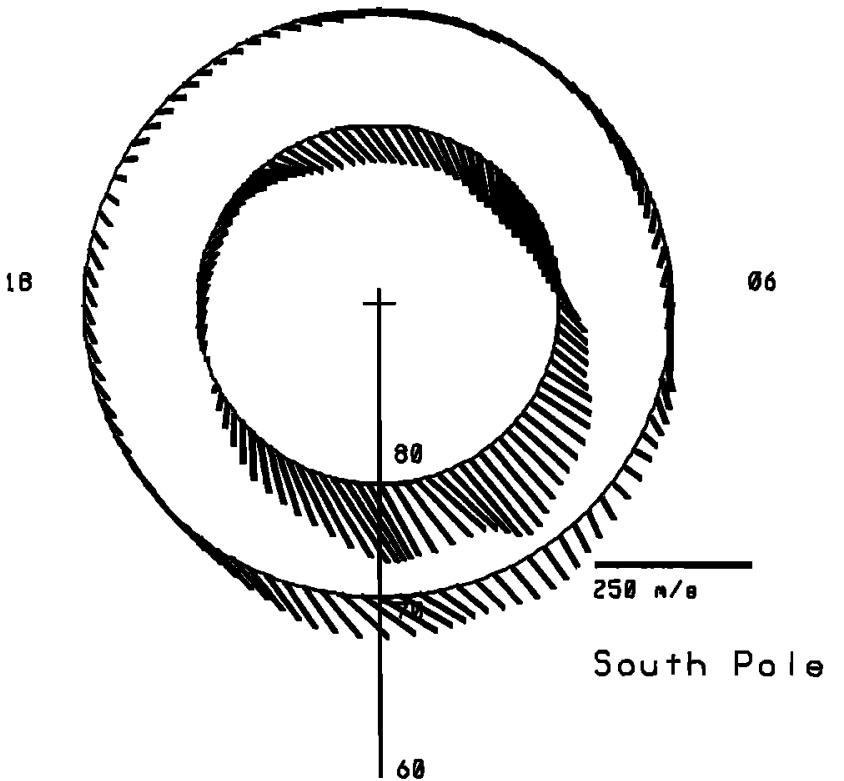


12

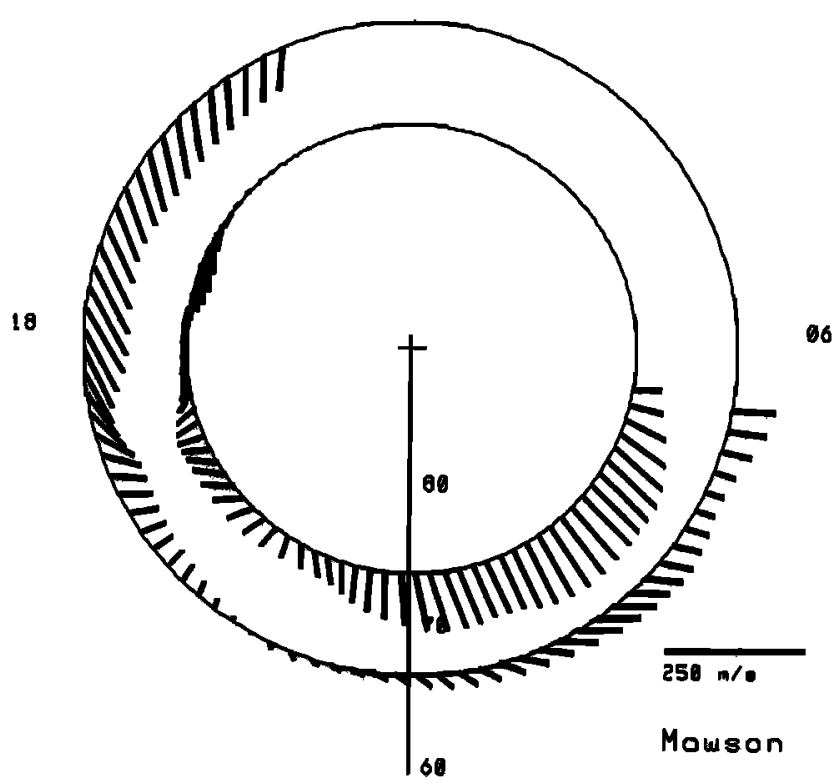

Figure 6. Polar plot of measured winds in the upper thermosphere at Mawson, averaged for the quiet period 920802 to 920804 . The center of this plot is at the geomagnetic pole, the circles are in geomagnetic latitude, and times are marked in MLT.

tent convergence in the meridional wind lasting for about 16 hours in which the wind looking poleward exceeded that looking equatorward. The maximum zonal wind was eastward (mean $75 \mathrm{~m} / \mathrm{s}$ ) in the evening twilight. Figure 6 shows the polar vector plot of the same data in geomagnetic coordinates. The winds were antisolar in the moming and evening, and the cross-polar jet, on the poleward side of the station, was about $100 \mathrm{~m} / \mathrm{s}$ at maximum near 0200 MLT and weaker, but slightly rotated eastward on the equatorward side. The lower panel in Figure 4 shows that, for Mawson, observed temperatures were similar to those at South Pole and a maximum appeared in the early aftemoon MLT.

\subsection{Halley}

Averaged observed geomagnetic meridional and zonal wind components for the days 920804,920806 , and 920807 are shown in Figure 7. A gap occurred in the record when operations were suspended during twilight at daylight. In a similar way to wind measurements from Mawson, they were spline-interpolated at half-hour intervals and rotated from the geographic directions of observation. The peak meridional wind of $130 \mathrm{~m} / \mathrm{s}$ was observed looking poleward near 1700 UT, which was just after magnetic midnight. This was presumed to correspond with the extension of the cross-polar jet in the geomagnetic latitude range $-57^{\circ}$ to $-65^{\circ}$. The peak wind observed looking equatorward at the same time was $50 \mathrm{~m} / \mathrm{s}$. Highly divergent meridional winds were observed during the early evening hours. A major characteristic of the meridional winds at Halley was that the diurnal variation looking poleward was considerably greater than that looking equatorward.

Peak zonal winds of $200 \mathrm{~m} / \mathrm{s}$ eastward were observed in evening twilight, the eastward observation being greater than the westward observation by $100 \mathrm{~m} / \mathrm{s}$. After magnetic midnight the zonal winds turned westward, continuing the diumal trend that zonal winds were consistent with antisolar flow. Figure 8 shows the polar vector plot

\section{Upper Thermospheric Winds at Halley}

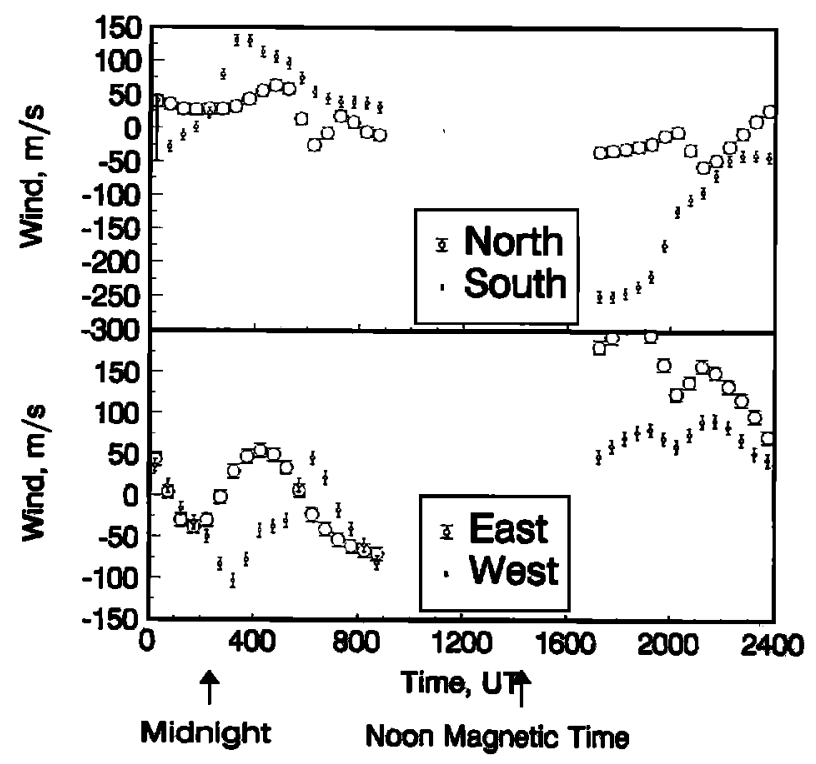

Figure 7. Wind components in the upper thermosphere measured at Halley, Antarctica, and rotated from observations in the geographic directions and plotted in geomagnetic coordinates. Averages were made for days in the quiet period 920802 to 920804 .

of the same data, indicating, how the early evening antisolar flow was part of the air parcel traffic into the high-latitude region while the midnight sector flow was part of the cross-polar jet exiting to lower latitudes. This figure also emphasizes the great differences in diumal variation which existed in the poleward direction compared to that equatorward. Halley was the only station which showed this effect.

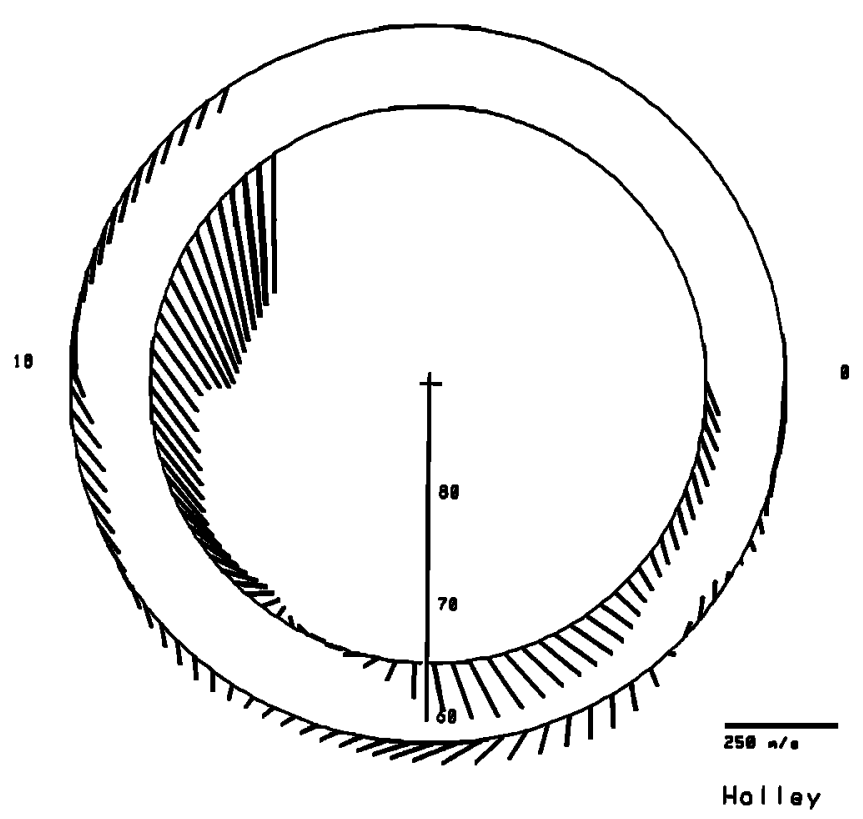

Figure 8. Polar plot of measured winds in the upper thermosphere at Halley, averaged for the quiet period 920802 to 920804 . The center of this plot is at the geomagnetic pole, the circles are in geomagnetic latitude, and times are marked in MLT. 


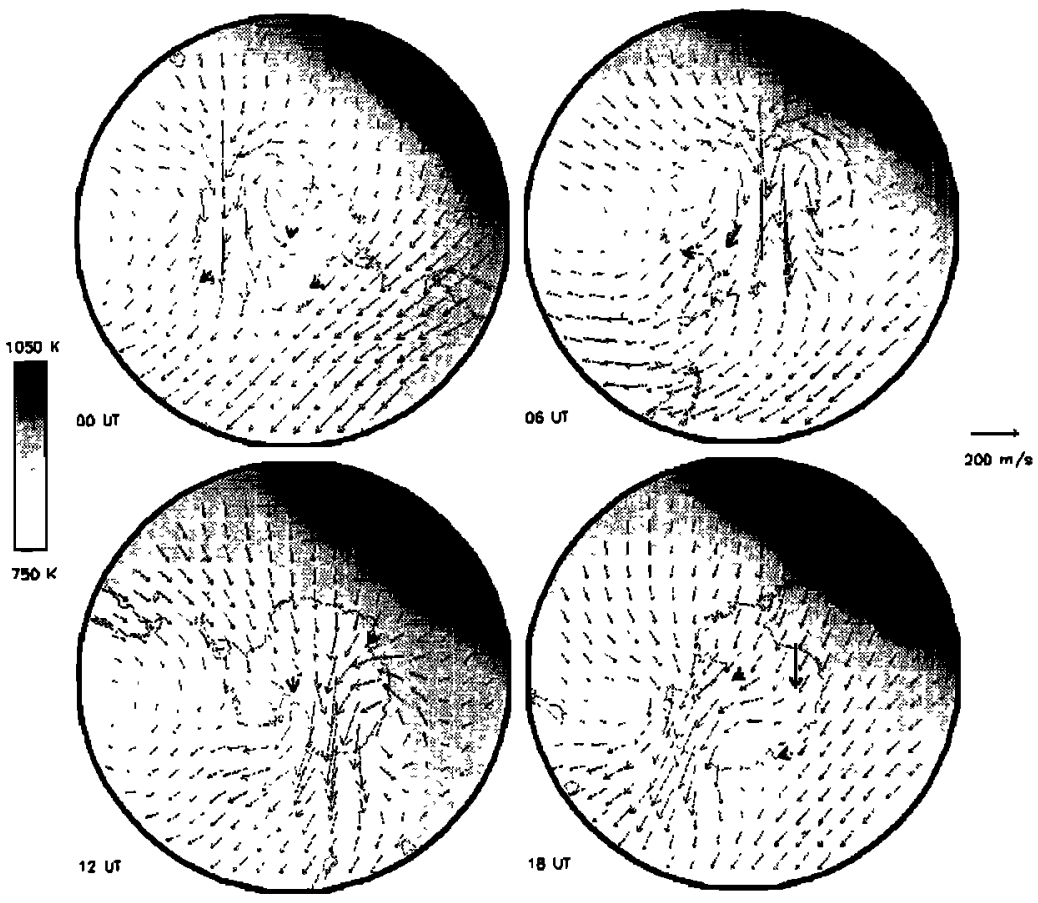

Figure 9. Polar plots of wind and temperature from the TIEGCM with wind observations superimposed. Proceeding from upper left to lower right, the times are 0000,0600,1200 and $1800 \mathrm{UT}$, respectively. Local noon is at the top of each plot and other times are located as for a clock with a 24 hour dial. Simulated winds appear as normal arrows, and spatial means of measured winds are shown by bold arrows located above the observatories. Simulated temperatures are shown in greyscale with reference to the scale bar on the left of the figure.

\section{TIEGCM Simulations}

The TIEGCM model was used to simulate the quiet time behavior of the upper thermosphere appropriate for comparison with the previously described data set. Several attempts were made to match the observations, and use was made of dynasonde and digisonde ionospheric profiles from Halley and Mawson in that process to calibrate the model ionosphere. Figure 9 shows four polar plots of simulated wind and temperature at $350 \mathrm{~km}$ altitude for 0000,0600 , 1200 , and $1800 \mathrm{UT}$ as well as the mean observations from each station at those times. Local solar noon is at the top of each polar plot with 1800 local solar time (LST) on the right side (three o'clock position). Wind vector measurements from each of the three stations are shown in bold and simulated winds in lighter arrows. Simulated temperatures are indicated by the grey shading and should be read with the aid of the shade bar on the left of the figure. There were three major persistent features in the simulated high latitude circulation system at all UT times. A cross-polar jet was continually found in the vicinity of the geomagnetic pole. An evening vortex was consistently found but with varying intensity. A large complex region of vortical flow bordered the morning side of the jet, extending well into the midlatitude zone. This region contained a small high latitude vortex at 0000 and 0600 UT but not at other times. Although the general pattern remained the same, it moved around the geographic pole with the diurnal cycle of the UT day and was modulated in strength with changes in ionospheric densities. The extremes of the UT effect, at the closest and farthest points of the geomagnetic pole from the Sun, occurred at 1600 and 1800 UT, respectively. The thermosphere was not sunlit except for a thin region of the Antarctic coast in the direction of 1200 LST. Hence the simulations on the dark side applied to conditions where the ionosphere-thermosphere coupling was strongly dependent on ion- ization occurring with the aurora and transport occurring by convection.

The plot for 0000 UT, at the top left of Figure 9, shows the crosspolar jet centered on a region just downstream from the geomagnetic pole with the predominant direction antisunward. At the time of this plot, the cross-polar jet flow went directly over Mawson. The simulation indicated a broad region of fast flow coming from the 1400 LST direction spread throughout the evening sector, the poleward boundary of which was sampled at Halley station. South Pole was on the geomagnetic poleward boundary of the dusk vortex. The simulation was in good agreement with observations at all stations. There was, however, a tendency for observed winds to be lower than the TIEGCM winds by up to $30 \%$.

At 0600 UT (Figure 9, top right) the simulation shows that the cross-polar jet began in sunlight at the coast of Antarctica. It reached its greatest velocity of $400 \mathrm{~m} / \mathrm{s}$ in the diurnal cycle at this time because of the higher electron density in the ionosphere and hence greater ion-neutral coupling. Well downstream from the jet, velocity vectors turned eastward making a track in a wide arc ending in a sunward direction. South Pole station lay on the morning side of the jet and sampled flow well away from the maximum velocity shown by the TIEGCM. Halley was downstream of South Pole, sampling air parcels which had turned eastward and were entering the equatorward part of the high-latitude morning vortex. We note here that the simulation indicated that there was a region of almost stagnant midlatitude flow equatorward of the high-latitude vortex. The high-latitude and midlatitude features each have vortex-like appearance but should be considered as separate phenomena. At 0600 UT the TIEGCM showed that Mawson was in the flow entering the polar cap on the dawn side but it was also in sunlight, preventing any observations being made. Simulated winds agreed well with measurements at South Pole but were overestimates at Halley. Tem- 
peratures decreased from a maximum of $1100 \mathrm{~K}$ at $-40^{\circ}$ geographic latitude at $1500 \mathrm{LST}$ in the sunlit hemisphere to a minimum of 850 $\mathrm{K}$ at $-75^{\circ}$ geographic latitude at $0300 \mathrm{LST}$ in the dark hemisphere. Although the South Pole temperature observations showed a maximum for the day near this time, no temperature maximum appeared in the nighttime high-latitude region on the simulation plot.

In the 1200 UT (Figure 9, bottom left) plot the TIEGCM showed that cross-polar jet had a maximum speed of $350 \mathrm{~m} / \mathrm{s}$. Air parcels in the jet were headed toward New Zealand and the Pacific Ocean. At this time, Mawson was under the flow entering the polar cap from the dusk side, and South Pole was still on the morning side of the jet but nearer the inflow region than at 0600 UT. Halley was in sunlight and not providing data. The observations from South Pole and Mawson stood in the convergent flow entering the cross-polar jet at this time. The TIEGCM agreed with directions of the winds entering the jet, but as in the 0000 UT case, the observed winds were found to be weaker than the simulation. Temperatures at Mawson were found to be highest near $1200 \mathrm{UT}$, which was expected by the model, since the station was closest to the global thermospheric temperature maximum at this time, and the local flow came directly from it. Interestingly, the TIEGCM plot suggests an interpretation for the subsequent rapid temperature fall at Mawson in that air parcel advection very quickly switched to a path from the nightside where temperatures were lower.

At 1800 UT (Figure 9, bottom right), the simulated cross-polar jet reached its maximum at the coast of Antarctica on its way toward Australia. Since the magnetic pole was farthest from the Sun, ion-neutral coupling was weaker except at locations where local auroral ionization or tongues of long-lived ionization patches crossed the polar cap. The maximum velocity of the simulated jet was now $310 \mathrm{~m} / \mathrm{s}$ but was not sampled by any of the three stations in this study. Halley was in the flow coming from the low-latitude pressure high near $1400 \mathrm{LST}$, much of which later converged to enter the cross-polar jet. The TIEGCM was in agreement with the magnitude of the Halley vector but differed by $30^{\circ}$ in direction. South Pole sampled air parcels of similar origin and nearby position to those measured at Halley but observed a much lower velocity. Although the vector direction in the simulation agreed with observation at South Pole, the magnitude was about $30 \%$ higher. Mawson lay near the equatorward edge of the eye of the evening vortex, where the winds were light and variable.

For South Pole the TIEGCM showed a diumal temperature maximum of $880 \mathrm{~K}$ near $1600 \mathrm{UT}$ and a minimum of $840 \mathrm{~K}$ near 0400 UT, which was quite different from the observations of $820 \mathrm{~K}$ and $900 \mathrm{~K}$ at the same times, respectively. The location of the hightemperature zone at South Pole suggests an association with the cross-polar jet. The diurnal variation for Mawson appears to have had the same amplitude and mean values as the TIEGCM, and no particular trends in temperature structure were found associated with the cross-polar jet of the evening vortex. The low temperature zone at Mawson near 1700 UT (2100 LST) appeared to be consistently colocated with the weak evening vortex. It appears that any connection between high thermospheric temperature and the cross-polar jet was confined to latitudes above $70^{\circ}$ invariant.

\section{Discussion}

Rather than present curves showing TIEGCM wind components versus observation, we prefer to discuss the examination of measurement and simulation in terms of the observations of the three principal structures, the cross-polar jet and the morning and evening vortices. The reason for this approach lies in the fact that the signa- ture of a vortex in terms of measured wind components along a line of sight is very sensitive to the precise details of the encounter. Differences between simulation and observation which occur in this way are not substantive in the study of high-latitude flow. Of more importance is the disposition and activity associated with each of the principal structures. The group of stations has some experience of the cross-polar jet, the evening vortex, and the morning vortex during each day. We proceed by discussing each in turn. Following that, we discuss the thermal behavior.

\subsection{The Cross-Polar Jet}

South Pole station observed the cross-polar jet to exit to lower latitudes with a speed of about $200 \mathrm{~m} / \mathrm{s}$ but with a direction towards 0200 MLT (compare Figure 3). The TIEGCM simulation agreed with the observed jet speed; however, the direction was somewhat westward of the simulated velocity (see Figures 3 and 9). The TIEGCM was also in agreement with the Mawson jet speed; however, in this case the directions were also in agreement, both being about the same angle on the east side of equatorward. Halley measured a jet of about $100 \mathrm{~m} / \mathrm{s}$ equatorward, which the model matches well. At all stations there was a strong tendency for the wind to slow down rapidly in the equatorward direction. The simulations showed a tendency for a minor stagnation region at the junction of the crosspolar flow and the broad fast flow in the late evening sector in the vicinity of 0000 UT. It seems reasonable to attribute the speed reductions observed at all three stations with a similar broad region of stagnation which persisted from 2200 UT to 0400 UT.

\subsection{Morning Vortex}

South Pole observed the edge of the moming vortex near 1200 UT each day. This was the location of a local minimum in wind speed observed in the late morning MLT. Observed velocities reduced $50 \mathrm{~m} / \mathrm{s}$ in magnitude and rotated westward. Mawson entered the morning vortex as dawn approached near 0600 MLT (0430 UT). Instead of showing antisunward flow due to the global thermospheric pressure gradient, the winds remained equatorward but decreased in magnitude. The TIEGCM showed that this was the direction of winds upon entry to the dawn vortex. At Halley the encounter with the morning vortex was closest near 0300 MLT (0600 UT). The meridional wind component decreased to zero, and the zonal component became westward in a similar way to the TIEGCM simulation. In Halley's case the encounter was with the lower latitude part of the complex morning vortical region.

\subsection{Evening Vortex}

The closest approach to the evening vortex at South Pole was near 2000 MLT (0000 UT), where the horizontal wind speed dropped to near zero while the vector rotated from westward through poleward to eastward. The TIEGCM showed the evening vortex centered on South Pole at this time. Experimentally, the rotation of the vector suggests that the vortex did not reach such high geomagnetic latitudes as were modeled in the TIEGCM. Mawson dwelled in the evening vortex from 1900 to 2300 MLT (1700 to $2100 \mathrm{UT}$ ) and perhaps longer. This was also the time when the evening vortex was weakest. The signature was not just weak winds but a sharp apparent convergence of flow in the zonal components suggestive of a rapid wind reduction or reversal in the region near the station. At 1800 UT the TIEGCM model placed the evening vortex overhead the Mawson station, providing a useful interpretation for this otherwise puzzling feature which has been found persistently in the ob- 
Upper Thermospheric Temperature $250 \mathrm{~km}$ altitude at South Pole Station

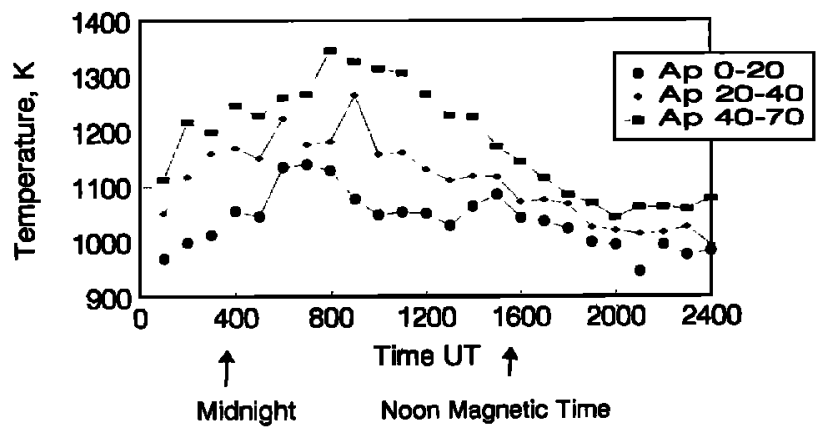

Figure 10. Upper thermospheric temperatures at South Pole station averaged for all directions and selected $A p$ ranges when F10.7 was between 200 and 250 .

servations. Halley, at $61^{\circ}$ invariant latitude, was never close to the evening vortex. Its closest approach was near $0200 \mathrm{MLT}$ (0000 UT), when it sampled the slow flow adjoining the global pressure gradient wind and the evening vortex.

\subsection{The Diurnal Temperature Variation at South Pole}

Mean diumal observed temperatures predicted by the TIEGCM for Mawson and South Pole were in substantial agreement with measurement. However, the diurnal variation predicted for South Pole shows the minimum near magnetic midnight and the maximum near magnetic noon. Figure 4 shows that observations indicated the opposite. This has been found to be a persistent trend at South Pole under all geomagnetic conditions [Smith and Hernandez, 1995] and opposite to the trend reported for very high geomagnetic latitudes in the northern hemisphere by McCormac et al. [1988] for Thule $\left(76.5^{\circ} \mathrm{N}, 68.8^{\circ} \mathrm{W}, 86^{\circ}\right.$ geomagnetic latitude). On the other hand, unpublished data from the Longyearbyen observatory $\left(78.2^{\circ} \mathrm{N}\right.$, $15.2^{\circ} \mathrm{E}, 75^{\circ}$ geomagnetic latitude), a northern hemisphere cusp station, show the same behavior as South Pole. The observations presented in this paper would suggest that there was a temperature maximum region in early postmidnight hours including $-75^{\circ}$ geomagnetic latitude. Figure 10 shows how the diurnal temperature variation changed with magnetic activity for $F 10.7$ between 200 and 250 . Since $F 10.7$ was lower for the present study period, the average temperatures were lower but the form of the diurnal variation was the same. The times of maximum and minimum temperatures were the same, within the time resolution of the plots, but the amplitude increased with increase in geomagnetic activity as represented by $A p$. At the highest levels of $A p$, the amplitude of the diurnal temperature variation reached $200 \mathrm{~K}$ with maximum temperature near 0400 MLT (0800 UT).

Another important aspect of the occurrence of the peak temperature near geomagnetic midnight is that this is the time when the cross-polar jet crossed the "path" of South Pole station. The accelerated air parcels in the cross-polar jet were hotter than other parcels which had been measured during the rest of the day. The rapid drop in temperature in Figure 4 between 2300 and 0100 UT occurred at about the right time to be the signature of leaving the heated thermosphere in the cross-polar jet. Since the acceleration of crosspolar jet parcels occurred by a combination of ion drag and pressure gradient (see, for example, Killeen and Roble [1984]) at least a portion of the process must have been accompanied by heating
[McCormac et al., 1988]. For a typical mean cross-polar speed of $300 \mathrm{~m} / \mathrm{s}$ and a distance of 30 degrees of latitude $(3000 \mathrm{~km})$ the residence time of an air parcel in the jet is about $10,000 \mathrm{~s}$, or about 3 hours. The time taken for an air parcel to be accelerated by the iondrag process at upper thermospheric heights is on the order of 1 hour, but may be a factor of 2 less or more, depending on conditions (see, for example, Rishbeth, [1972]). For assumed quasi-stationary conditions, it is likely that an air parcel will have almost reached the ion drift velocity after traversing the polar cap. Using that assumption, one may estimate the Joule heat associated with the polar cap crossing by considering the heating rate constant during the acceleration period and zero thereafter.

The mean Joule heating rate in the polar cap during acceleration may be estimated using the formula $Q\left(\mathrm{~W} / \mathrm{m}^{2}\right)=\Sigma E^{2}$, where $\Sigma$ is a mean Pedersen conductance in the polar cap (say, 2 mhos) and $E$ is a mean electric field in the polar cap (say $15 \mathrm{mV} / \mathrm{m}$ ). For an acceleration time of 1 hour, the total Joule heat will be $1.6 \mathrm{~J} / \mathrm{m}^{2}$. If this heat has been generated only above $150 \mathrm{~km}$, at thermal equilibrium the expected temperature rise would be given by $\Sigma E^{2} /(1.5 n k H)$ where $n$ and $H$ are the number density and scale height at $150 \mathrm{~km}$, respectively, and $k$ is the Boltzmann constant. For an exospheric temperature of $1100 \mathrm{~K}$, the MSIS model estimates number density at 150 $\mathrm{km}$ to be $4 \times 10^{16} \mathrm{~m}^{-3}$ and $H$ to be $24 \mathrm{~km}$. Hence it is found that a temperature rise of $80 \mathrm{~K}$ could occur by Joule heating while the cross-polar jet accelerated. On this basis of crude estimation, it appears reasonable to find about $100 \mathrm{~K}$ temperature difference between air parcels entering the cross-polar jet stream on the dayside and exiting on the nightside at $75^{\circ}$ invariant latitude. Alternatively, it may be said that it is not unreasonable to associate the measured temperature difference between dayside and nightside with heating incurred by the ion drag portion of the acceleration of the crosspolar jet.

Examination of the plots in Figure 9 shows no tendency in the TIEGCM simulations to show increased temperatures on the nightside compared to the dayside of the polar cap within $15^{\circ}$ of the geomagnetic pole. Since the Joule heating processes are included in the model, it can be presumed that the simulated heating effect was not sufficient to provide the observed temperature rises. On the other hand, a negative temperature gradient existed from early aftemoon to early morning. Hence the simulated heating effect in the polar cap might have appeared less obvious because of the need to reverse the global diumal temperature gradient before creating a temperature maximum on the nightside.

The difference in temperature variation between data and simulation can also be discussed in terms of the model inputs which affect the heating at high latitudes. Reduction of heating in the vicinity of the cusp could be achieved by reduction of cusp extent. Increased nighttime auroral zone heating at $F$ region heights may be obtained with softer electron fluxes, more plasma heating in the polar cap or inclusion of an ac component in the electric field which would raise the Joule heating. Adjustments to input parameters such as these may improve the agreement. However, consideration of such modifications has not been included here, since the present paper has concentrated on examination of the simulations with the usual prescription for these input parameters.

As was noted in section 2, within 5 hours of a flat temperature profile being observed at Mawson crossing of the jet, a temperature maximum was observed $8^{\circ}$ farther poleward at Mawson. This observation suggests that the high temperature seen at the geomagnetic latitude of South Pole was confined to a region which did not extend to the Mawson latitude. If this had been represented on the TIEGCM plot, a zonally oriented region of heated thermosphere 
would have been found on the nightside not extending equatorward beyond $70^{\circ}$ geomagnetic latitude. The cause of this may be associated with a loss of the heating source and the mixing which occurs with a fan-out of the jet as it proceeds to lower latitudes (see Figure 9).

\section{Conclusions}

The persistent features of the polar upper thermospheric wind field, and the cross-polar jet, and the moming and evening vortices were encountered in the observations from the group of stations. In general, comparison of observed wind directions with the TIEGCM simulation was very good, although the experimental wind magnitudes were often overestimated as much as $30 \%$. The TIEGCM predicted the cross-polar jet in generally good agreement with observation. The encounters of Halley and Mawson with the vortices caused apparent convergences in the observed wind which were explained in terms of the wind patterns simulated by the TIEGCM. South Pole did not pass as close to the evening vortex as was predicted by the TIEGCM.

Observations of temperatures from South Pole showed that there was a local temperature minimum on the dayside of the polar cap and a local maximum on the nightside near $75^{\circ}$ geomagnetic latitude. The temperature variations from the TIEGCM calculations were $180^{\circ}$ out of phase by comparison with observation. Interpretation of the observed diurnal temperature variation suggests that it could be associated with heat generated during the acceleration process of the cross-polar jet. On the basis of a simple calculation, it appears that a temperature increase of $100 \mathrm{~K}$ between the input and output of the polar jet acceleration process is reasonable. Although the production of Joule heat was included in the TIEGCM it did not produce an effect of the magnitude observed. It can also be suggested that measurement of the diurnal temperature amplitude at South Pole station would lead to an estimate of the Joule heating during the acceleration processes in the cross-polar jet.

Acknowledgements. This work was supported by NSF grants OPP9316163, ATM9300274, and ATM9416710 and by grants from the Australian Research Council and the Australian Antarctic Science Advisory Committee. Antarctic stations at Halley and Mawson are supported by the British Antarctic Survey and the Australian Antarctic Division, respectively.

The Editor thanks A.G. Burns and another referee for their assistance in evaluating this paper.

\section{References}

Burns, A. G., T. L. Killeen, W. Deng, G. R. Carignan, and R. G. Roble, Geomagnetic storm effects in the low- to middle-latitude upper thermosphere, J. Geophys. Res., 100, 14,673-14,691, 1995.

Codrescu, M. V., R. G. Roble, and J. M. Forbes, Interactive ionosphere modeling: A comparison between TIGCM and ionosonde data, J. Geophys. Res., 97, 8591-8600, 1992.

Crickmore, R. I., J. R. Dudeney, and A. S. Rodger, Vertical thermospheric winds at the edge of the auroral oval, J. Atmos. Terr. Phys., 53, 486-492, 1991

Crowley, G., B. A. Emergy, R. G. Roble, H. C. Carlson Jr., J. E. Salah, V. B. Wickwar, K. L. Miller, W. L. Oliver, R. G. Bumside, and F. A. Marcos, Thermospheric dynamics during September 18-19. 1984, 2, Validation of the NCAR thermospheric general circulation model, J. Geophys. Res., 94, 16,945-16,959, 1989.

Deng, W., T. L. Killeen, A. G. Burns, R. G. Roble, J. A. Slavin, and L. E Wharton, The effects of neutral inertia on ionospheric currents in the high-latitude thermosphere following a geornagnetic storm, J. Geophys. Res., 98, 7775-7790, 1993.

Forbes, J. M., R. G. Roble, and F. A. Marcos, Magnetic activity dependence of high-latitude thermospheric winds and densities below $200 \mathrm{~km}, J$. Geophys. Res., 98, 13,693-13,702, 1993.

Hemandez, G., and R. G. Roble, Thermospheric nighttime neutral temperature and winds over Fritz Peak Observatory: Observed and calculated solar cycle variation, J. Geophys. Res., I00, 14,647-14,659, 1995.

Hernandez, G., R.W. Smith, R.G. Roble, J. Gress, and K.C. Clark, Thermospheric dynamics and the South Pole, Geophys. Res. Lett., 17, 1255-1259, 1990.

Jacka, F., Application of Fabry-Perot spectrometers for measurement of upper atmospheric temperatures and winds, Handbook for MAP 13, 19-40, ICSU Scientific Committee on Solar-Terrestrial Physics (SCOSTEP), Urbana, Illinois, 1984.

Killeen, T. L., and R. G. Roble, An analysis of the high latitude thermospheric wind pattern calculated by a thermospheric general circulation model, 1, Momentum forcing, J. Geophys. Res., 89, 7509-7522, 1984.

Lu, G., A. D. Richmond, B. A. Emery, and R. G. Roble, Magnetosphereionosphere-thermosphere coupling: Effect of neutral winds on energy transfer and field-aligned current, J. Geophys. Res., 100, 19,643-19,659, 1995.

McCormac, F. G., T. L. Killeen, A. G. Burns, and J. W. Meriwether, Polar cap diurnal temperature variations: Observations and modelling, $J$. Geophys. Res., 93, 7466-7477, 1988.

Richmond, A. D., E. C. Ridley, and R. G. Roble, A thermosphere/ionosphere general circulation model with coupled electrodynamics, Geophys. Res. Lett., 19, 601-604, 1992.

Rishbeth, H., Thermospheric winds and the $F$ region: A review, J. Atmos. Terr. Phys., 34, 1-47, 1972.

Rishbeth, H., B. Jenkins, and R. J. Moffett, The F-layer at sunrise, Ann. Geophys., 13(4), 367-374, 1995.

Roble, R. G., The polar lower thermosphere, Planet. Space Sci., 40, 271297, 1992.

Smith, R. W., and G. Hernandez, Upper thermospheric temperatures at South Pole, Adv. Space Res., 16(5), 31-39, 1995.

Smith, R. W., J. W. Meriwether Jr., G. Hernandez, D. Rees, V. Wickwar, O. de la Beaujardiere, and T. L. Killeen, Mapping the wind in the polar thermosphere: A case study within the CEDAR program, Eos Trans. AGU, $70,161,168-169,1989$.

Sojka, J. J., R. W. Schunk, and G. L. Wrenn, A comparison of $f_{0} F_{2}$ obtained from a time-dependent ionospheric model with Argentine Islands' data for quiet conditions, J. Atmos. Terr. Phys., 50, 1027-1039, 1988.

M. Conde and R. W. Smith, Geophysical Institute, University of Alaska, Fairbanks, AK 99775-7320. (e-mail: conde@giuaf.gi.alaska.edu; bblw@ giuaf.gi.alaska.edu)

R. Crickmore, Winfrith Technology Centre, Winfrith, Nerburgh, Dorchester, Dorset DT3 8X, England, United Kingdom.

P. L. Dyson, School of Physics, LaTrobe University, Bundoora, Victoria 3083, Australia. (e-mail: p.dyson@latrobe.edu.au)

G. Hemandez, Graduate Program in Geophysics, AK-50, University of Washington, Seattle, WA 98195. (e-mail: hernandez@geophys. washington.edu)

M. Jarvis, British Antarctic Survey, Madingly Road, Cambridge CB3 0ET, England, United Kingdom. (e-mail: m.jarvis@bas.ac.uk)

R. G. Roble, High Altitude Observatory, National Center for Atmospheric Research, P.O. Box 3000, Boulder, CO 80307-3000. (e-mail: roble@near.ucar.edu)

(Received June 27, 1997; revised November 12, 1997; accepted November 13, 1997.) 